\title{
Editorial
}

\section{Neutrino Physics}

\author{
Jose Bernabeu, ${ }^{1,2}$ Gian Luigi Fogli, ${ }^{3,4}$ Arthur B. McDonald, ${ }^{5}$ and Koichiro Nishikawa ${ }^{6}$ \\ ${ }^{1}$ Department of Physics, University of Valencia, C/Dr. Moliner 50, Burjassot, 46100 Valencia, Spain \\ ${ }^{2}$ Instituto de Física Corpuscular, Universitat de Valencia-Consejo Superior de Investigaciones Cientificas (UV-CSIC), \\ C/Catedrático José Beltrán 2, Paterna, 46980 Valencia, Spain \\ ${ }^{3}$ Dipartimento Interateneo di Fisica "Michelangelo Merlin", Università di Bari, Via Amendola 173, 70126 Bari, Italy \\ ${ }^{4}$ Istituto Nazionale di Fisica Nucleare, Sezione di Bari, Via Orabona 4, 70126 Bari, Italy \\ ${ }^{5}$ Department of Physics, Engineering Physics and Astronomy, Stirling Hall, Queen's University, Kingston, ON, Canada K7L 3N6 \\ ${ }^{6}$ Institute of Particle and Nuclear Studies, High Energy Accelerator Research Organization (KEK), 1-1 Oho, \\ Tsukuba 305-0801, Ibaraki, Japan
}

Correspondence should be addressed to Jose Bernabeu; jose.bernabeu@uv.es

Received 20 January 2013; Accepted 20 January 2013

Copyright (C) 2013 Jose Bernabeu et al. This is an open access article distributed under the Creative Commons Attribution License, which permits unrestricted use, distribution, and reproduction in any medium, provided the original work is properly cited.

Neutrino physics has provided very impressive progress in our understanding of particle physics during recent years. These advances are of primary relevance for a better description of neutrino properties, and presumably they also contain profound implications in the general picture of fundamental interactions and their connections with astrophysics and cosmology. The central role of neutrinos is even more evident when considering that neutrino masses require physics beyond the standard theory. At present, however, we do not have a clear picture of such new physics and its energy scale.

Recently the connecting mixing angle $\theta_{13}$ between the atmospheric and solar sectors has been determined to be relatively large. This opens exciting possibilities for upcoming neutrino oscillation experiments addressing fundamental questions like the neutrino mass hierarchy and the search for $\mathrm{CP}$ violation in the lepton world. The present volume of contributions contains original research articles as well as review papers able to stimulate the continuing advance of knowledge in this field and the prospects and strategies for the next future. These articles describe all facets of this broad field, being experimentally developed with both terrestrial accelerators and astroparticle sources, studies in underground laboratories and including the theoretical scenario.

The paper "The nature of massive neutrinos" by S. T. Petcov reviews the compelling experimental evidence for oscillations of solar, reactor, atmospheric, and accelerator neutrinos implying the existence of 3-neutrino mixing in the weak-charged lepton current. The properties of massive
Majorana neutrinos and of their various possible couplings are discussed in detail. Two models of neutrino mass generation with massive Majorana neutrinos, the type I see-saw and the Higgs triplet model, are briefly reviewed. The problem of determining the nature, Dirac or Majorana, of massive neutrinos is considered. The predictions for the effective Majorana mass in neutrinoless double beta decay in the case of 3-neutrino mixing and massive Majorana neutrinos are summarised. The open questions and the main goals of future research in the field of neutrino physics are outlined.

The paper "Neutrinoless double-beta decay" by A. Giuliani and A. Poves discusses the current state and future prospects for experiments and theory for measurements of this unique process which has not yet been clearly observed. Neutrinoless double beta decay is energetically allowed for a limited set of nuclei and can only take place if the neutrino is a Majorana particle and has a finite mass. The half life for the process is in excess of $10^{24}$ years and is very hard to measure. However, a measurement can provide a sensitive determination of an effective mass which is a combination of masses and mixing parameters for the three neutrino mass eigenstates. Combining this with oscillation measurements could provide absolute masses for all three eigenstates.

The paper "Atmospheric neutrinos" by T. Kajita discusses production and detection of atmospheric neutrinos. Atmospheric neutrino experiments were the first ones to find neutrino oscillation. The zenith-angle and energy-dependent deficit of muon neutrino events was found in such a way that neutrino oscillations between muon neutrinos and tau 
neutrinos explain these data well. This article discusses atmospheric neutrino experiments and the future possibilities of the neutrino oscillation studies with them.

In the paper "Neutrino propagation in matter", by M. Blennow and A. Y. Smirnov, the authors describe in detail the effects of neutrino propagation in the matter of the Earth, relevant for experiments with atmospheric and accelerator neutrinos aiming at the determination of the neutrino mass hierarchy and CP violation. The results of semianalytic descriptions of flavor transitions for the cases of small density perturbations in the limit of large densities and for small density widths are included. In particular the possibility of identifying the neutrino mass hierarchy with atmospheric neutrinos through multimegaton scale detectors having low energy thresholds and with future accelerator experiments is explored.

The paper "Solar neutrinos" by V. Antonelli et al. discusses the past history, current status and future prospects for experiment and theory of neutrinos produced by nuclear reactions in the sun. Measurements of these solar neutrinos have provided fundamental information about neutrino properties and about solar models. There are prospects for extending this information in future measurements described in the paper.

The paper "Solar neutrino observables sensitive to matter effects" by H. Minakata and C. Peña-Garay discusses the interaction of solar neutrinos with matter in the sun. Such neutrino propagation in matter is described quite well by the Mikheyev-Smirnov-Wolfenstein (MSW) theory and the paper discusses present and future measurements that can test this theory in more detail, as well as providing information on neutrino properties and solar models via matter interactions.

The article "Reactor neutrinos" by S.-B. Kim et al. reviews the status and the results of reactor neutrino experiments. Middle and long baseline oscillation experiments provided very recently the most precise determination of the neutrino mixing angle $\theta_{13}$ and measurements of $\theta_{12}$ and $\Delta \mathrm{m}^{2}$. This paper also provides an overview of the upcoming experiments and of the projects under development, including the determination of the neutrino mass hierarchy and the possible use of neutrinos for society, for non proliferation of nuclear materials and geophysics.

The paper "Long-baseline neutrino oscillation experiments" by G. Feldman et al. is a review of long-baseline accelerator neutrino oscillation experiments, including all experiments performed to date and the projected sensitivity of those currently in progress. Accelerator experiments have played a crucial role in the confirmation of the neutrino oscillation phenomenon and in precision measurements of the parameters. Evidence for electron neutrino appearance has recently been obtained, opening the door for determining the mass hierarchy and the CP violating phase: some of the last unknown parameters of the standard model extended to include neutrino mass.

The paper "Sterile neutrino fits to short baseline neutrino oscillation measurements" by J. M. Conrad et al. reviews short-baseline oscillation experiments as interpreted within the context of one, two, and three sterile neutrino models associated with additional neutrino mass states in the $1 \mathrm{eV}$ range. It is shown that while fitting short-baseline data sets to a $(3+3)$ model, defined by three active and three sterile neutrinos, yields the highest quality overall, it still finds inconsistencies with the MiniBooNE appearance data sets. These results motivate the pursuit of further short-baseline experiments, such as those reviewed in this paper.

The interesting subject of sterile neutrinos is discussed for a different energy scale in the article "Search for GeV-scale sterile neutrinos responsible for active neutrino masses and baryon asymmetry of the universe" by D. S. Gorbunov et al. In particular, the authors provide a motivation for the existence of three new neutral Majorana particles (sterile neutrinos), associated with simultaneous solution of the problems of neutrino masses and oscillations, of baryon asymmetry of the universe, and of dark matter. A new beam-target experiment is proposed to search for new physics beyond the Standard Model below the Fermi scale, which may lead to discovery of these right-handed partners of the known neutrinos.

The paper "Current direct neutrino mass experiments" by G. Drexlin et al. discusses the measurement of neutrino mass through very sensitive experiments examining the distortion of beta decay spectra near the end point via various techniques. The present status and future prospects for techniques using electrostatic retardation or low-temperature bolometers form the principal discussion, with other more unusual techniques included for possible future measurements.

One important input in the determination of the absolute neutrino masses is an accurate Q-value of the beta or electron capture decay. This can be done, from the difference of the masses of the parent and the daughter nuclei, by means of Penning traps. In the paper "A quantum sensor for neutrino mass measurements" by J. M. Cornejo and D. Rodriguez, a novel device with unprecedented accuracy and sensitivity is presented. The method can also be applied to the search of the resonant enhancement mechanism for the neutrinoless double electron capture candidates.

In the paper "Neutrino mass from cosmology" by J. Lesgourgues and S. Pastor, the authors discuss the important role played by neutrinos in the evolution of the Universe, modifying some of the cosmological observables. In this contribution the main aspects of cosmological relic neutrinos are summarized. The precision of present cosmological data can be used to learn about neutrino properties, in particular their mass, providing complementary information to beta decay and neutrinoless double beta decay experiments. The authors show how the analysis of current cosmological observations, such as the anisotropies of the cosmic microwave background or the distribution of large-scale structure, provide an upper bound on the sum of neutrino masses of order $1 \mathrm{eV}$ or less, with very good perspectives from future cosmological measurements which are expected to be sensitive to neutrino masses well into the sub-eV range.

In "Neutrinos and Big Bang nucleosynthesis" by G. Steigman, the major role of neutrinos in the early universe is discussed in detail, including effects on abundances of light elements formed at this time and the relationship of these abundances to the total number of neutrino types. 
The paper "The Era of kilometer-scale neutrino detectors" by F. Halzen and U. Katz discusses the scientific missions of IceCube and KM3NeT instruments, which include such varied tasks as the search for sources of cosmic rays, the observation of Galactic supernova explosions, the search for dark matter, and the study of the neutrinos themselves. Identifying the accelerators that produce the Galactic and extragalactic cosmic rays has been a priority mission of several generations of high-energy gamma-ray and neutrino telescopes; success has been elusive so far. Detecting the gamma-ray and neutrino fluxes associated with cosmic rays reaches a new watershed with the completion of IceCube, the first neutrino detector with sensitivity to the anticipated fluxes. In this review, the authors first revisit the rationale for constructing kilometer-scale neutrino detectors and subsequently recall the methods for determining the arrival direction, energy, and flavor of neutrinos. The architecture of the IceCube and KM3NeT detectors is described.

The measurement of electron antineutrinos from the decay of Uranium, Thorium and other elements in the earth can be used to determine the abundances of these elements in the crust and mantle and their contribution to the total heat flow in the earth. The past, present, and future experimental and theoretical status is discussed in detail in "Geoneutrinos" by $\mathrm{O}$. Šràmek et al., including the importance of these measurements for our understanding of the geophysics of the earth.

The paper "Neutrino Yukawa textures within type-I seesaw" by B. Adhikary and P. Roy presents a classification of possible texture zeros in the Dirac neutrino mass matrix of the see-saw mechanism, given the basis where the charged lepton mass matrix and the right-handed Majorana neutrino mass matrix are both diagonal. Such a study is important in view of current experimental progress in determining the active (light) neutrino mixing angles, in particular a relatively large value of $\theta_{13}$. Accordingly, the masses and Majorana phases of ultralight neutrinos are predicted within definite ranges and the rate of the neutrinoless double beta decay, though generally below the reach of planned experiments, is seen to approach it in some parametric regions.

In the paper "Leptogenesis in the Universe" by C. S. Fong et al., the main aspects of leptogenesis are considered, that is, the class of scenarios in which the cosmic baryon asymmetry originates from an initial lepton asymmetry related to the decay of heavy sterile neutrinos in the early Universe. The authors describe why leptogenesis is an appealing mechanism for baryogenesis, by reviewing motivations, basic ingredients, and specifically related effects. Leptogenesis in supersymmetric scenarios is also addressed, as well as some other popular variations of the basic leptogenesis framework.

In the paper "Electromagnetic properties of neutrinos" by C. Broggini et al., the authors discuss the main theoretical aspects and experimental effects of neutrino electromagnetic properties. A general description of the electromagnetic form factors of Dirac and Majorana neutrinos is given. Then, the theory and phenomenology of the magnetic and electric dipole moments is presented, summarizing the experimental results and the theoretical predictions. They discuss also the phenomenology of a neutrino charge radius and radiative decay. Finally, the theory of neutrino spin and spin-flavor precession in a transverse magnetic field is developed, summarizing its phenomenological applications.

In "The results of a search for the neutrino magnetic moment in the GEMMA experiment" by A. Georgievich Beda et al., an upper limit for the neutrino magnetic moment is presented from a measurement at the Kalinin Nuclear Power Plant (KNPP) with the GEMMA spectrometer.

There is a large interest in neutrino interactions in the intermediate energy region, motivated by the need in neutrino oscillation experiments to reduce systematic errors. The subject is reviewed in the paper "Recent developments in neutrino/antineutrino-nucleus interactions" by J. G. Morfín et al. They discuss recent inclusive measurements in the lower energy region and then concentrate on exclusive states in the increasing of the mass of the hadronic system, giving in this way an exhaustive review of the current theoretical and experimental situation of neutrino interactions in this energy region.

Neutrinos from supernovae are important probes not only of the currently unknown supernova mechanism but also of neutrino properties. In the paper "Charged-current neutrino-nucleus scattering off the even molybdenum isotopes", E. Ydrefors and J. Suhonen compute the cross-sections of the above processes for an extensive set of neutrino energies, thus obtaining the nuclear responses to supernova neutrinos by folding these cross-sections with a Fermi-Dirac distribution.

The article "Prospects for neutrino oscillation physics" by S. Pascoli and T. Schwetz reviews the phenomenology of neutrino oscillations, focusing on subleading effects, which will be the key towards the goals of the determination of the type of the neutrino mass hierarchy and the search for $\mathrm{CP}$ violation in the lepton sector. Starting from a discussion of the present determination of three-flavour oscillation parameters, an outlook of the potential of near term oscillation physics is given, as well as on the long-term program towards possible future precision oscillation facilities. Accelerator driven longbaseline experiments as well as nonaccelerator possibilities from atmospheric and reactor neutrinos are discussed.

The article "Future long-baseline neutrino facilities and detectors" by M. Diwan et al. discusses the next generation neutrino detectors and neutrino beam facilities. These facilities will address two aspects: the fundamental properties of neutrinos like mass hierarchy, mixing angles, and the $\mathrm{CP}$ phase, as well as low-energy neutrino astronomy with solar, atmospheric, and supernova neutrinos. A new detector naturally allows for major improvements in the search for nucleon decay. A next generation neutrino observatory needs a huge detector, which in turn has to be installed in a new international underground laboratory capable of hosting such a huge detector.

Jose Bernabeu Gian Luigi Fogli Arthur B. McDonald Koichiro Nishikawa 

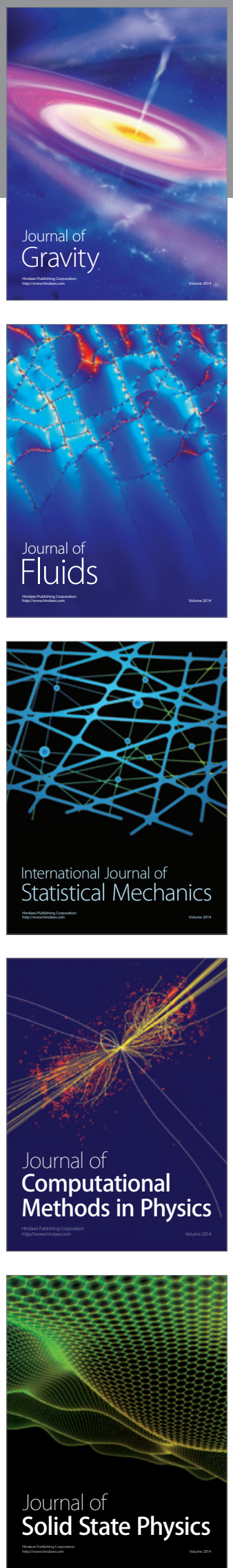

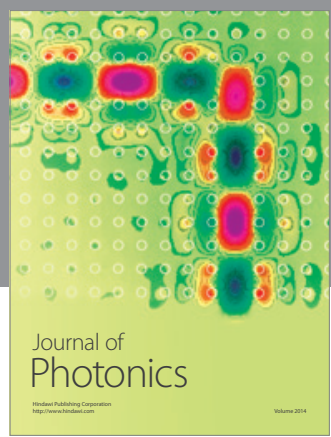

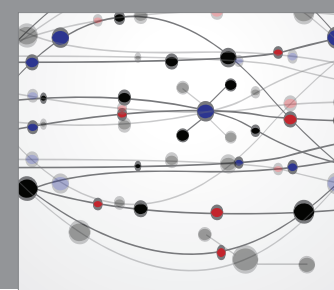

The Scientific World Journal

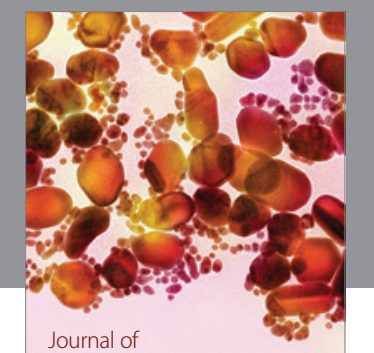

Soft Matter
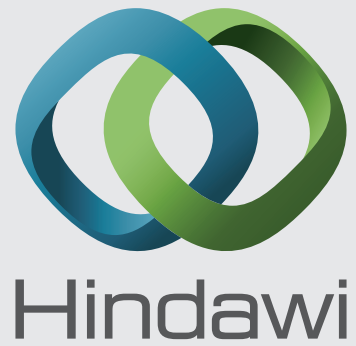

Submit your manuscripts at

http://www.hindawi.com
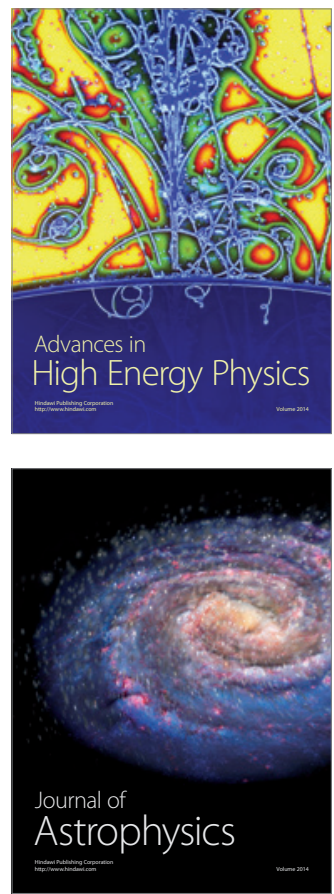
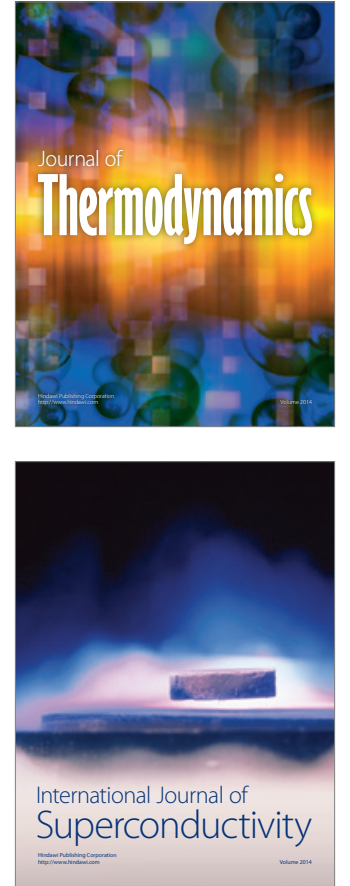
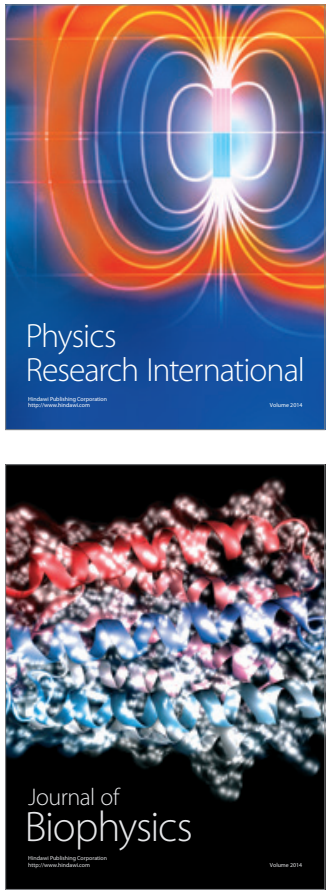
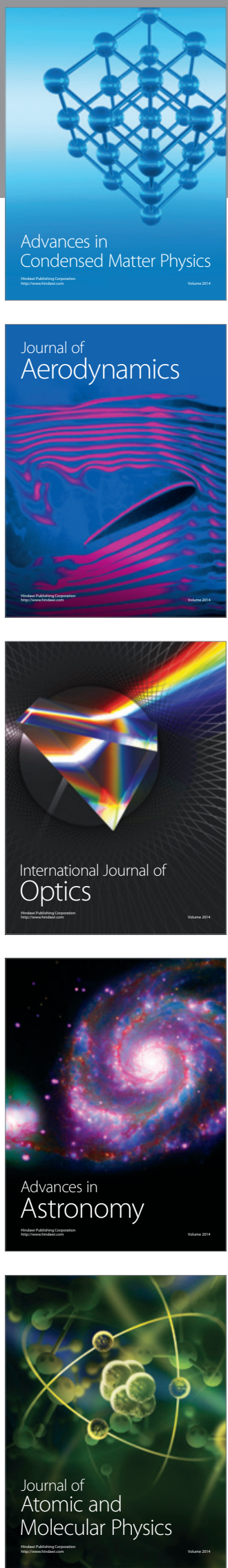